\title{
Identification and replication of RNA-Seq gene network modules associated with depression severity
}

Trang T. Le ${ }^{1,2}$, Jonathan Savitz ${ }^{2,3}$, Hideo Suzuki ${ }^{2,4}$, Masaya Misaki ${ }^{2}$, T. Kent Teague, 5,7, Bill C. White $\mathbb{0}^{8}$, Julie H. Marino ${ }^{9}$, Graham Wiley $^{10}$, Patrick M. Gaffney ${ }^{10}$, Wayne C. Drevets ${ }^{11}$, Brett A. McKinney ${ }^{1,8}$ and Jerzy Bodurka ${ }^{2,12}$

\begin{abstract}
Genomic variation underlying major depressive disorder (MDD) likely involves the interaction and regulation of multiple genes in a network. Data-driven co-expression network module inference has the potential to account for variation within regulatory networks, reduce the dimensionality of RNA-Seq data, and detect significant geneexpression modules associated with depression severity. We performed an RNA-Seq gene co-expression network analysis of mRNA data obtained from the peripheral blood mononuclear cells of unmedicated MDD $(n=78)$ and healthy control $(n=79)$ subjects. Across the combined MDD and HC groups, we assigned genes into modules using hierarchical clustering with a dynamic tree cut method and projected the expression data onto a lower-dimensional module space by computing the single-sample gene set enrichment score of each module. We tested the singlesample scores of each module for association with levels of depression severity measured by the Montgomery-Åsberg Depression Scale (MADRS). Independent of MDD status, we identified 23 gene modules from the co-expression network. Two modules were significantly associated with the MADRS score after multiple comparison adjustment (adjusted $p=0.009,0.028$ at 0.05 FDR threshold), and one of these modules replicated in a previous RNA-Seq study of $\operatorname{MDD}(p=0.03)$. The two MADRS-associated modules contain genes previously implicated in mood disorders and show enrichment of apoptosis and B cell receptor signaling. The genes in these modules show a correlation between network centrality and univariate association with depression, suggesting that intramodular hub genes are more likely to be related to MDD compared to other genes in a module.
\end{abstract}

\section{Introduction}

RNA-Seq is a transcriptome profiling technique that uses next-generation sequencing to provide a sensitive, quantitative measurement of RNA abundance or gene expression. Challenges associated with the RNA-Seq approach include both technical limitations (e.g., tissue heterogeneity and batch effects) and statistical concerns (e.g., over dispersion and multiple hypothesis testing). Furthermore, major depressive disorder (MDD) is a

\footnotetext{
Correspondence: Brett A. McKinney (brett-mckinney@utulsa.edu) or Jerzy Bodurka (jbodurka@laureateinstitute.org)

${ }^{1}$ Department of Mathematics, The University of Tulsa, Tulsa, OK, USA

${ }^{2}$ Laureate Institute for Brain Research, Tulsa, OK, USA

Full list of author information is available at the end of the article.
}

complex phenotype involving systems of interacting genes, and single-gene associations of expression have not reached genome-wide significance. While these approaches have provided biological insights and identified candidate biomarkers associated with some neurological diseases $^{1,2}$, network and gene-gene interaction approaches may enrich the variable space to better predict or characterize the genomic architecture of more complex phenotypes ${ }^{3}$.

Co-expression network techniques for detecting coordinated gene expression changes at a gene set (modular) level have potential power to provide novel insights into the genetic architecture of psychiatric disorders ${ }^{4,5}$. A module can be understood as a collection of genes that 


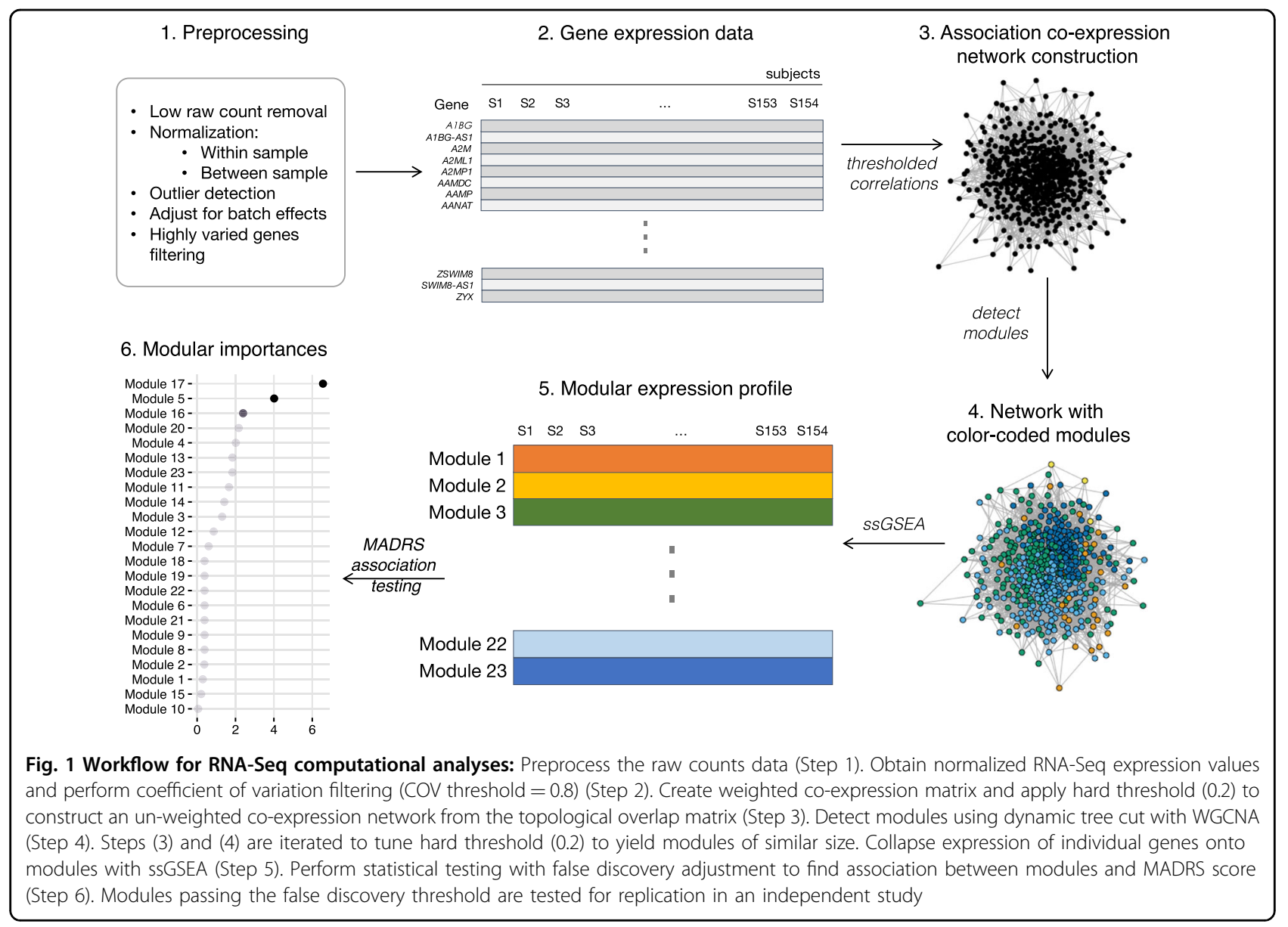

are highly interconnected (e.g., by co-expression) and, thus, more likely to share a similar biological function ${ }^{6}$. Modular analysis also helps alleviate the multiple hypothesis testing problem inherent in RNA-Seq data and may be more robust than single-gene investigation. Clustering thousands of genes into pathway-sized modules and collapsing these genes onto single statistical units significantly reduces the number of hypotheses to be tested. Combined with statistical learning methods, as well as meta-analyses of existing databases, modular analyses of co-expression networks have been conducted in several studies to identify groups of differentially expressed genes in schizophrenia ${ }^{7-9}$, autistic spectrum disorder ${ }^{10}$, Alzheimer's disease ${ }^{11}$, and $\mathrm{MDD}^{12}$.

Initial genome-wide association studies (GWAS) of MDD had limited success at finding significant variants due to the contribution of many loci with small effect sizes as well as the heterogeneous characteristics of MDD and the complex interaction between genetic variation and environmental factors ${ }^{13}$. More recently, many small, but significant, main effect loci have been identified through the accumulation of extremely large samples ${ }^{14,15}$. Similarly, it has been difficult to identify significant single- gene effects at the expression level from RNA-Seq of MDD. In Mostafavi's RNA-Seq study of 922 subjects, only 29 genes were found to have significant association with MDD status at the relaxed FDR threshold of 0.25 , but sets of top genes were significantly enriched for the IFN $\alpha / \beta$ signaling pathway ${ }^{16}$. Combining a modular approach with meta-analysis of 11 transcriptome studies of postmortem brains, Chang et al. identified a transcriptome module of 88 genes based on consistency with GWAS results for MDD, other neuropsychiatric disorders, and brain function ${ }^{12}$. This meta-module is enriched for genes that encode proteins implicated in neuronal signaling and structure.

Modularity is a ubiquitous feature of biological systems ${ }^{17}$ and genes within modules tend to be functionally related $^{18,19}$, which may help us find regulatory genes that affect disease risk along with direct disease-related genes. Thus, in the present study, we applied a data-driven approach to detect depression gene modules (DGMs), which are co-expression modules associated with depression phenotypes. Because our analytical approach is sensitive to weak individual effects and takes biological interactions among genes into account, it can potentially 
reveal biological effects that are neglected in univariate models. Using RNA-Seq from peripheral blood, we constructed a co-expression network for the combined MDD and healthy control (HC) subjects and created hierarchical clusters of similar size using the dynamic tree cut from the weighted gene co-expression networks analysis (WGCNA) tool ${ }^{20}$. We then projected each subject's genelevel expression onto a lower-dimensional space of modules using single-sample gene set enrichment analysis (ssGSEA) approach ${ }^{21,22}$.

The resulting 23 modular expression profiles were not conditioned on the phenotype and thus may be used as predictor variables in a greatly reduced hypothesis space. We applied false discovery rate adjusted linear regressions to each modular expression profile to identify modules that are associated with subject's depression severity characterized by the Montgomery-Åsberg Depression Scale (MADRS). We then explored the relation of several genes in these significant modules to the clinical phenotype, MDD, along with other psychiatric disorders based on the extant literature. Of the two modular expression profiles that survived multiple hypothesis testing, one module (DGM-5) replicated in an independent data set.

\section{Methods and materials}

The co-expression network module analysis involves multiple steps to obtain gene set predictors of MDD (Steps 1-6, Fig. 1). In this section, we provide details of the RNA-Seq preprocessing of the raw count data, normalization of expression values, and variation filtering (Steps 1 and 2). We describe our iterative approach to module construction using a hard threshold of the coexpression matrix and the topological overlap matrix (Step 3), combined with clustering by the dynamic tree cut algorithm (Step 4). Steps 3 and 4 are iterated with a grid of hard thresholds to obtain modules of similar size. We reduce the hypothesis space by collapsing the expression of individual genes onto these modules (Step 6) and test these module features for association with MADRS score with false discovery rate adjustment.

\section{Subjects}

Participants between the ages of 18 and 55 years were recruited from the clinical services of the Laureate Psychiatric Clinic and Hospital (LPCH) and media advertisements in the Tulsa metropolitan area, Oklahoma. A total of $N=160$ subjects, including 80 subjects who met DSM-IV-TR criteria for MDD (52 females, mean age = $33 \pm 11$ ) and $80 \mathrm{HCs}$ who showed no history of any major psychiatric disorder in a first-degree relative (41 females, mean age $=31 \pm 10$ ), participated in the study. However, because one MDD subject's expression data were corrupted and two additional subjects (one MDD and one $\mathrm{HC}$ ) were outliers, their data were excluded from the analyses (see below for details). The diagnosis of MDD was established using the Structural Clinical Interview for DSM-IV-TR Axis I Disorders (SCID-I/NP; 1 January 2010) and confirmed by an unstructured interview with a psychiatrist. Exclusion criteria included the use of psychotropic medications for at least 3 weeks prior to study entry, major medical or neurological illness, psychosis, traumatic brain injury, and a history of drug/alcohol abuse within 1 year. All subjects gave written informed consent to participate in our study and received financial compensation.

The present study was approved by the Western Institutional Review Board, and it was conducted according to the principles expressed in Declaration of Helsinki. All participants gave written informed consent to participate and received financial compensation.

\section{Materials}

The clinician-administered Montgomery-Åsberg Depression Rating Scale (MADRS; Williams \& Kobak, 2008) was used to rate the severity of depressive symptoms. In clinical trials of major depressive disorder, the ten-item diagnostic questionnaire MADRS is accepted by the FDA and other health authorities as valid and reliable rating instruments for obtaining the primary outcome measure of antidepressant treatment efficacy ${ }^{23}$.

\section{Steps 1 and 2. RNA-Seq data generation and processing}

Morning blood samples were obtained from the participants, and peripheral blood mononuclear cells (PBMCs) were isolated using cell preparation tubes. We quantified RNA expression obtained from frozen (PBMCs) by analyzing complementary DNA derived from the PBMCs with RNA-Seq. Following initial quality-control steps, sequencing libraries were generated using the Illumina Truseq Stranded mRNA with library prep kit according to the manufacturer's protocol. Sequencing was performed on an Illumina Hiseq 3000 instrument with paired-end $150 \mathrm{bp}$ reads. Samples were sequenced to an average depth of 30 million reads and RNA integrity number of 8.6 per sample. RNA-Seq measures gene expression by sequencing, yielding the abundance of each transcript present. After gene-level transcripts were computed from transcriptomic sequencing, the sequencing reads are aligned and mapped to individual exons. We used RefSeq for the cDNA alignment. The total number of read counts was obtained per gene from the mRNA expression. Normalization of the gene counts was performed with conditional quantile normalization (CQN), which accounts for differences in library size and also adjusts for GC content and gene length ${ }^{24}$. These normalized values were used for subsequent analyses. The RNA-Seq raw counts preprocessing steps involved: (i) removal of genes with low counts (threshold defined below) and 
normalization, (ii) outlier detection, (iii) batch effect correction, and (iv) high coefficient of variation (COV) filtering of genes. Briefly, analyses included autosomal genes with $\geq 15$ individuals with $\geq 2-7$ reads, depending on the library size. Then, we applied an angle-based outlier $(\mathrm{ABO})$ detection $^{25}$ to remove samples with exceptionally small $A B O$ factor. Batch effect was adjusted with the function "removeBatchEffect" from the $\mathrm{R}$ package "limma" ${ }^{26}$ (Fig. S2). Reasoning that expression values that differ greatly across subjects are likely due to technical variability $^{27}$, we excluded genes with (COV) larger than 0.8 to obtain genes whose expression values were roughly consistent across samples. Details on data generation and preprocessing are provided in Supplements 1, 2. Preprocessed RNA-Seq data are available upon request to corresponding authors.

\section{Steps 3 and 4. Gene co-expression network construction and module identification}

We used an iterative procedure to identify the module predictors for association testing with depression severity. We first built a co-expression network by calculating the correlation of the pairwise gene expression, applied a hard threshold to the network and then computed the network's topological overlap matrix (TOM) dissimilarity between the genes (Fig. 1, Step 3). We used the new TOM distance matrix from the hard threshold to construct a hierarchical tree and used dynamic tree cutting to construct modules (Step 4). Steps 3 and 4. (Fig. 1) were repeated for a grid of hard thresholds to obtain a final hard threshold $(0.2)$ that yields similar module sizes (mean size of 200 genes $^{28}$ ). In the optimal weighted network, we removed edges with correlation values below a threshold of 0.2. Our motivation was to find cluster sizes that were relatively similar in size and with a large enough number of genes for ssGSEA to be effective. Gene set enrichment often tests modules of size 200 genes, and having similar module sizes (Fig. S3) help alleviate potential module-size bias in ssGSEA. We note that we did not detect correlation between module-size and statistical significance of ssGSEA module associations with depression phenotype. We obtained 23 collections of genes (modules) with similar connectivity in the coexpression network. Slight variation of the hard threshold value does not have a meaningful effect on the number of modules.

To perform the hierarchical clustering, we used unsigned weighted correlation networks analysis ${ }^{20}$ (WGCNA), which has been used in a variety of fields (e.g., cancer and brain imaging analysis ${ }^{29}$ ). Specifically, we measured dissimilarity between pairs of expression values, created a dendrogram of genes, and identified modules from the different levels of similarity structure. We used a dynamic tree cut clustering method ${ }^{30}$ to identify modules from the TOM matrix of this network of normalized gene expression values. The WGCNA tool includes a dynamic tree cut method that provides a flexible dendrogram cutting mechanism that is effective at detecting nested modules.

\section{Step 5. Projection of module gene sets onto lower- dimensional feature space}

We generated normalized enrichment profiles for each gene cluster using single-sample gene set enrichment analysis (ssGSEA) ${ }^{21,22}$. Similar to the notion of eigengene in WGCNA ${ }^{20}$, ssGSEA calculates an enrichment profile of modules in each subject based on individual expression values in the modules. However, instead of using a principal component analysis, ssGSEA is based on the cumulative distribution of the ranked expression values. Particularly, ssGSEA assigns a sample-level enrichment score to a gene module by rank-normalizing the expression values and comparing the empirical cumulative distribution of these ranks inside and outside that module. The scaled module's score of a sample represents the degree to which its genes are coordinately up- or downregulated within that sample. Consequently, within a particular sample, the expression profile in the higherdimensional space of genes is projected onto a lowerdimensional space of modules, yielding a smaller set of new variables that helps reduces the hypothesis space's dimension and is more biologically interpretable.

One of our goals was to test module hypotheses in an independent data set. We felt the ssGSEA would provide a more reproducible mechanism than eigengene for collapsing a set of genes onto a predictor variable. The ssGSEA method can be applied to an independent expression data set directly without recomputing a correlation matrix and the eigengenes. Furthermore, we did not strictly follow the WGCNA protocol to create modules. Rather than optimize a soft threshold power based on the degree of distribution, we optimized a hard threshold cutoff based on similar module sizes.

\section{Step 6. Testing gene module features for association with depression severity}

The modules' enrichment profiles were then considered as explanatory variables in the linear models to estimate each participant's scaled MADRS score. In addition to these enrichment scores, the design matrix also included sex, age, BMI, and batch as covariates. Further, because smoking status is known to be associated with depres$\operatorname{sion}^{31}$ and may confound gene expression ${ }^{32,33}$, we also considered an additional model where smoking status is added as a covariate. Moreover, although the majority of participants are Caucasian, the data set also contains 
Table 1 Characteristics of the sample

\begin{tabular}{|c|c|c|c|c|}
\hline Variable & All $(N=157)$ & $\operatorname{MDD}(n=78)$ & $\mathrm{HC}(n=79)$ & $t$ or $x^{2}$ \\
\hline Age (years) & $32(1)$ & $33(10)$ & $31(10)$ & $1.40(155)$ \\
\hline Sex & & & & 2.93 \\
\hline (Female/male) & $91 / 66$ & $51 / 27$ & $40 / 39$ & $(1)$ \\
\hline \multicolumn{5}{|l|}{ SRA } \\
\hline Caucasian & 120 & 59 & 61 & \multirow[t]{6}{*}{$1.09(5)$} \\
\hline African-American & 12 & 6 & 6 & \\
\hline Native American & 4 & 3 & 1 & \\
\hline Native Hawaiian/Pacific Islander & 2 & 1 & 1 & \\
\hline Asian American & 4 & 2 & 2 & \\
\hline Other & 15 & 7 & 8 & \\
\hline \multicolumn{5}{|l|}{ Occupational status } \\
\hline Employed full time & 55 & 25 & 30 & \multirow[t]{8}{*}{$7.62(7)$} \\
\hline Employed part time & 19 & 11 & 8 & \\
\hline Homemaker & 5 & 1 & 4 & \\
\hline Full-time student & 34 & 11 & 23 & \\
\hline Unemployed less than 6 months, but expects to work & 7 & 4 & 3 & \\
\hline Unemployed 6 months or more, but expects to work & 1 & 1 & 0 & \\
\hline Unemployed 6 months or more and does not expect to work & 1 & 1 & 0 & \\
\hline Other & 2 & 1 & 1 & \\
\hline \multicolumn{5}{|l|}{ Educational status } \\
\hline Some high school & 3 & 1 & 2 & \multirow[t]{6}{*}{$10.39(5)$} \\
\hline High school graduate & 11 & 8 & 3 & \\
\hline Some college/technical school & 62 & 32 & 30 & \\
\hline College graduate & 37 & 12 & 25 & \\
\hline Masters or above & 10 & 2 & 8 & \\
\hline Other & 1 & 0 & 1 & \\
\hline \multicolumn{5}{|l|}{ Smoking status } \\
\hline Non-smoker & 111 & 46 & 65 & \multirow[t]{2}{*}{$4.52^{*}(1)$} \\
\hline Smoker & 14 & 10 & 4 & \\
\hline $\mathrm{BMI}$ & $28.1(6.43)$ & $29.3(6.81)$ & $26.9(5.85)$ & $2.33^{*}(155)$ \\
\hline MADRS & $11.7(11.76)$ & $22.2(7.99)$ & $1.8(2.45)$ & $21.49^{* *}(150)$ \\
\hline
\end{tabular}

Values enclosed in the parenthesis represent standard deviations (under "All," "MDD," and "HC") or degrees of freedom (under " $t$ or $X^{2 \prime}$ ). The variables of SRA, occupational status, and educational status contained missing values

$H C$ healthy controls, SRA self-reported ancestry, BMI body mass index, MADRS total score on Montgomery-Åsberg Depression Rating Scale ${ }^{*} p<0.05 ;{ }^{* *} p<0.01$

other self-reported ancestries (SRAs). As a sensitivity analysis, we tested our hypotheses on a subset of the data with only Caucasian participants to examine whether the results were confounded by multiple SRAs. The $p$ values obtained from the analysis are corrected based on Benjamini-Hochberg's procedure ${ }^{34}$. Genes in modules with false discovery rate (FDR) adjusted $p$ values $<0.05$ were designated as differentially expressed in aggregate. We also search for the enriched genes among the genes within each significant module using GeneAnalytics ${ }^{35}$ and VarElect ${ }^{36}$ of the GeneCard Suites (http://www.genecards. org/) for additional interpretation. 


\section{Network centrality and individual gene's importance in discriminating phenotypes}

In a secondary univariate analysis, we investigated the individual effect of genes by conducting logistic regressions of the diagnosis phenotype (MDD/HC) on each of the 5912 genes and adjusting the $p$ values according to the Benjamini-Hochberg procedure ${ }^{34}$. We also computed eigenvector centrality for each of the 5912 genes based on the co-expression network. Centrality analysis approximates the relative importance of genes based on their connectivity within the network structure: a gene with higher centrality is more influential than a low-centrality gene. The simplest centrality metric is degree centrality, which counts the number of connections a specific gene has with other genes. In this analysis, we computed the eigenvector centrality, a variant of degree centrality that takes into account the importance of neighboring genes. Within the most significant modules, we then examined the relationship between each gene's centrality and its individual importance, measured as the negative log of its adjusted $p$ value:

$$
s_{i}=-\log \left(p_{i}\right) .
$$

We find that the centralities of genes within statistically significant modules are more correlated with their univariate diagnosis statistical association compared to genes in other modules.

\section{Results}

According to Chi-square test or $t$-test, there was no difference in age, sex, SRA, occupational status, and educational status between the MDD and HC groups (Table 1). The MDD group showed significantly higher BMI and, as expected, more severe depressive symptoms (based on MADRS score) than the HC group. Therefore, even though BMI does not significantly correlate with MADRS $(p=0.123)$, we controlled for BMI in our subsequent analyses to ensure our results were not confounded by BMI.

Figure 1 shows the overall workflow for RNA-Seq data analyses. Out of 19,968 identified genes 12,049 genes with a low count were removed. The remaining 7919 significant counts are then normalized and used as inputs to the angle-based outlier $(\mathrm{ABO})$ analysis, an outlier detection method that is robust for high-dimensional data (Supplement 2-ii). We also removed two outlier samples (one MDD and one $\mathrm{HC}$ ) with distinctly small $\mathrm{ABO}$ factors $(<0.001$, Fig. S1). We note that, if included in the analyses, these two samples would have had exceptionally high TMM normalization factors. In addition, 2007 expression values with high variability (calculated by coefficient of variation) were also filtered out. As a result, input to the gene co-expression network construction is a $\log \mathrm{CPM}$ matrix of dimension 157 samples $\times 5912$ genes. We note that the size of this filtered data set is similar to that of other gene expression studies ${ }^{8,28,37}$. Our iterative thresholding and dynamic branch cut of the co-expression network (Steps 3 and 4, Fig. 1) results in 23 modules. The number of genes in each module ranges from 86 to 746 genes.

After correcting for multiple hypothesis testing, we find that two modules' enrichment profiles, DGM-17 $(\beta=$ 15.4 , se $\left.=4.21, p_{\text {raw }}=0.000352\right)$ and DGM-5 $(\beta=-5.31$, $\left.\mathrm{se}=1.75, p_{\text {raw }}=0.00284\right)$, are significantly associated with MADRS score (Fig. S4). When tested on only 120 samples of Caucasian subjects, despite the reduction in power, these two modules remain significantly associated with this measure of depression severity $\left(p_{\text {raw }}=0.0024\right.$ and 0.0032 ). We concluded that the results were robust to SRA. Furthermore, when smoking status was added as a covariate in the linear model, DGM-17 ( $\left.p_{\text {raw }}=0.000395\right)$ and DGM-5 $\left(p_{\text {raw }}=0.00244\right)$ still showed statistically significant associations with MADRS. The results are similar likely because no participants in the data set were heavy smokers. However, because the Bayesian Information Criterion of this model is larger than that of the original model, we presented the result from the original model without smoking status.

DGM-17 and DGM-5 contain 109 and 291 genes, respectively, including VRK2, OPRM1, and TCF7L2 in DGM-17; AKT1, CREB1, CALB1, FAS, FKBP4, FOXP3, HDAC5, and PDE6C in DGM-5, which, as we discuss below, are components of pathways potentially related to mood disorders. Comparing this approach to the traditional individual significance of genes on diagnosis phenotypes, we found DGM-17 and DGM-5 contain significantly more top genes compared to other modules (hypergeometric test results of observing $x_{i}$ genes from module $i$ in the most 100 significant genes are shown in Table S2). Moreover, within these two significant modules, genes' global centralities are positively correlated with its statistical association with diagnostic status (Fig. 2). This high correlation between univariate gene significance and network centrality implies that genes with high centrality in DGM-17 and DGM-5 tend to be highly correlated with diagnosis status.

\section{Replication in previous RNA-Seq study of MDD}

We used the RNA-Seq study by Mostafavi et al. ${ }^{16}$ as a replication set to test for association with MDD of the significant modules, DGM-17 and DGM-5, from our current study. This independent data set consists of RNA-Seq measurements of 15,231 genes in 463 MDD cases and 452 controls. Of the 291 genes in module DGM-5 and 109 genes in module DGM-17 (Supplements 6, 7), we found 238 and 72 genes in Mostafavi's study that belong to these two modules, respectively. We applied ssGSEA on these genes to obtain the enrichment scores of 915 subjects for 


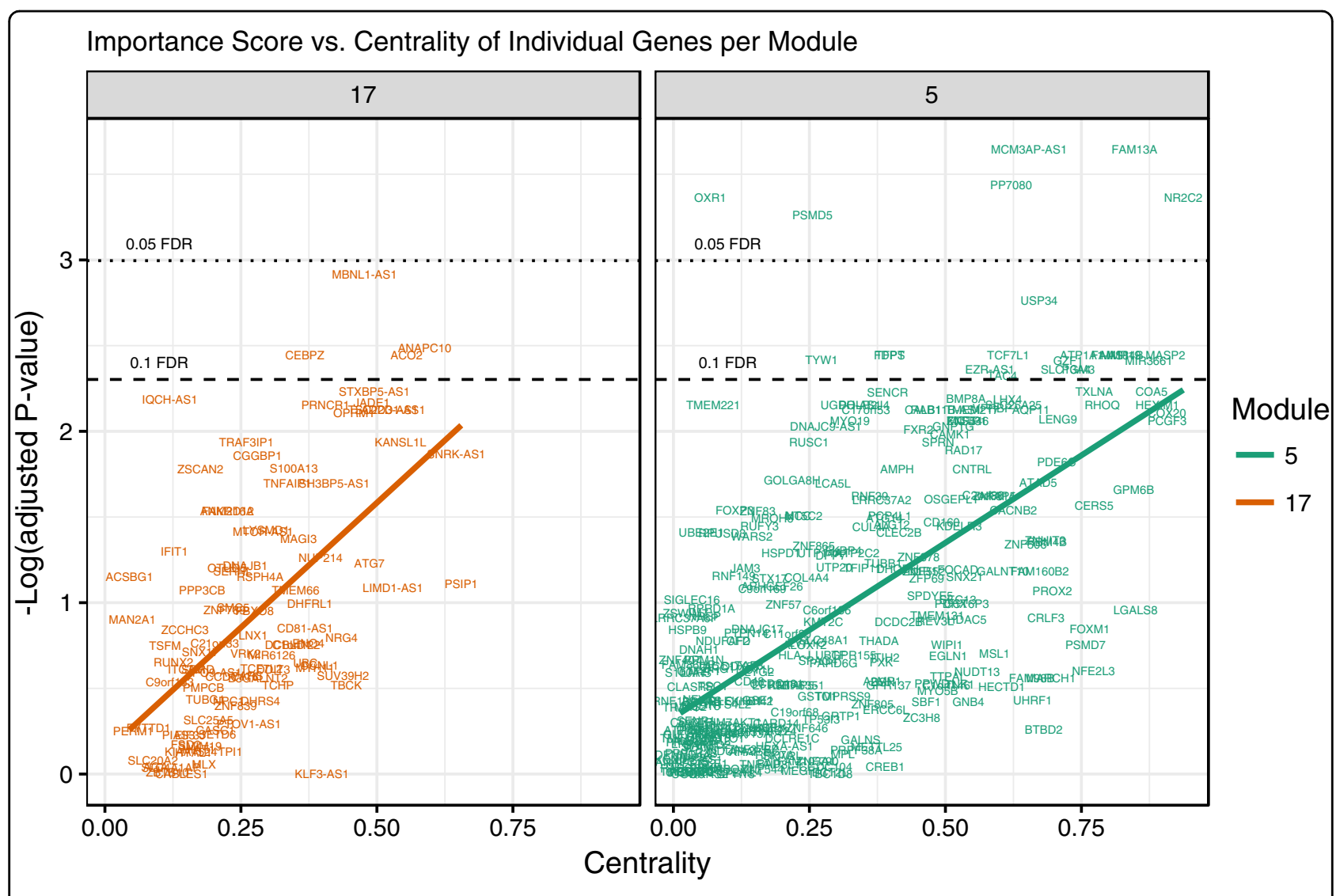

Fig. 2 Plot of individual importance vs. eigenvector centrality of genes in DGM-17 and DGM-5. "LOC" genes are not shown. Significant correlation observed between genes' individual phenotypic and network importance. $R_{(\mathrm{DGM}-17)}^{2}=0.3421 ; R_{(\mathrm{DGM}-5)}^{2}=0.3782$

the two modules. Because MADRS score is not reported in this independent data set, we alternatively ran a logistic regression of the diagnosis phenotype $(\mathrm{MDD} / \mathrm{HC})$ on the module's enrichment score, including sex, age, and BMI as in the original modular regression. Even though we only found $82 \%$ overlap of module DGM-5's genes with the Mostafavi data set, DGM-5's enrichment score was shown to be significantly associated with the diagnosis status in this independent data set ( $p$ value $=0.033)$. We found no significant association of DGM-17's enrichment value with the diagnosis phenotype in the replication set. However, this may be explained by the fact that more than a quarter of the genes in the original, already relatively small, module DGM17 are not present in the replication data set due to the difference in low-abundance filtering thresholds.

\section{Discussion}

We employed a novel combination of approaches to RNA-Seq data obtained from a cohort of depressed and healthy individuals that led to the replication of a depression gene module in a two-stage analysis. Some of these approaches include enforcing similar module sizes to guide co-expression network thresholding and gene set variation analysis to collapse genes onto modular units of analysis to reduce multiple hypothesis testing. Most gene expression studies have used individual genes as the unit of analysis for differential expression between phenotypes.

Module-based analysis is a sensitive technique to detect weak, but coordinated, gene expression changes at a module level. A related limitation of this approach is that summarizing the score for a module to one value, whether by ssGSEA, eigengene or other dimension reduction techniques, results in the loss of information at the single gene level. However, this technique reduces the high dimensionality of the hypothesis space by clustering thousands of genes into a manageable number of modules of interacting genes that may share similar biological functions. In the lower-dimensional variable space, statistical learning methods can be applied to identify gene modules that are significantly associated with depression severity without overfitting. Thus, we argue that a genemodule approach based on expression networks is a useful statistical model of the genetic architecture of complex diseases such as depression, in which multiple interacting homeostatic systems are affected ${ }^{38}$. 
The two statistically significant modules (after FDR adjustment) contain candidate genes for MDD and related disorders. Several genes in module DGM-5 include HDAC5 and CREB1 whose expression has been reported to be altered in MDD patients ${ }^{39}$. The histone methylation processes in which HDAC5 participates have also been implicated across different psychiatric disorders ${ }^{40}$. Linkage of variation in CREB1, the cyclic AMP response element-binding protein gene, to anger expression and treatment outcome in MDD patients ${ }^{41,42}$ as well as gender-specific susceptibility for $\mathrm{MDD}^{43,44}$ has been reported. CREB1 is also considered one of the important targets of antidepressants ${ }^{45}$. FOXP3, an intracellular marker for regulatory $\mathrm{T}$ cells (Tregs), has shown decreased expression level in depressed patients compare to $\mathrm{HC}$ group ${ }^{46}$, while our group previously reported increased circulating numbers of Tregs in MDD versus $\mathrm{HC}^{47}$. FOXP3 also plays an essential role in maintaining homeostasis of the immune system, one of the pathways that have significant association with aggregate psychiatric disorders ${ }^{40}$. Variation in FAS, a gene involved in $\mathrm{T}$ cell activation and apoptosis, is associated with antidepressant prognosis $^{48}$. A significant increase in FAS expression is also observed in depressed patients ${ }^{49}$.

Another noteworthy gene in module DGM-17 is OPRM1 because of its association with depression symptoms through interaction with stressful life events ${ }^{50}$. Alteration of opioid neurotransmission has also been observed in MDD patient $^{51}$. Additional gene-level information in the significant modules, DGM-5 and DGM-17, is summarized in Table 2 based on relevance to mood disorders from the literature. We found more mood disorder-related genes in the literature for DGM-5 than DGM-17, which may explain the fact that DGM-5 replicated while DGM-17 did not. However, we acknowledge that many of the genes found in the mood disorder literature have not been well replicated because, in part, MDD is a complex disorder of heterogeneous etiology. This complexity is a potential motivation for modular approaches that accumulate the coordinated variation of genes to detect gene modules related to depression. Module DGM- 5 also contains more genes than DGM-17; however, we did not find evidence of module-size bias, finding no correlation between module size and statistical significance of modules (results not shown). Another reason that DGM-17 did not replicate may be due to the lower overlap of genes in the replication data set $(83 \%$ for DGM-5 vs $73 \%$ for DGM-17).

In addition to containing several candidate genes, DGM-5 and DGM-17 show enrichment ( $q$ value 0.2 ) for several pathways involving immune function (Table 3). The enrichment of the apoptosis pathway in DGM-5 suggests a genetic signature involving brain regionspecific volume reduction due to cell loss in $\mathrm{MDD}^{52,53}$. The enriched PI3K/AKT activation pathway is involved in apoptosis and plays a role in mRNA translation of type I interferon-dependent genes ${ }^{54}$. The viral protein R (VPR) pathway, enriched in DGM-17, is involved in the induction of apoptosis in proliferating cells and B cell signaling. The DMG-5 module contains the binding protein for VPR (VPRBP in Supplement 7), which suggests additional overlap of the function of these two modules.

The apoptosis signal in the blood expression may originate from the brain (e.g., neuronal death due to apoptosis) and/or from other sources of cellular stress (e.g., activated $\mathrm{T}$ cells). The detection of the apoptosis signal in DGM-5 suggests the signal may be brain derived. One may strengthen the evidence for brain-derived apoptosis by testing for the association of expression of apoptosis genes with brain volumetric variation. This hypothesis could be tested in a whole-brain approach or a more targeted region-of-interest approach, conditioning on MDD status, and adjusting for age.

As a secondary analysis, we compared the co-expression network centrality of each gene with the statistical significance of its univariate effect on MDD status. Within the most significant modules, we found a positive correlation between a gene's centrality and the statistical significance of the gene's differential expression. In other words, "hub" genes in these top modules are potentially more predictive of the diagnosis phenotype compared to other genes that have lower centrality in the modules. In addition to the cumulative variation of genes within modules, using information related to the centrality of genes may improve the discovery of MDD-related genes and further limit the number of hypothesis tests. Hub genes in significant modules also may make it easier to identify biologically meaningful genes ${ }^{55}$.

One of the limitations of our study is the relatively small sample size. However, the dimensionality reduction, multiple test adjustment, and replication in a previous RNA-Seq MDD study adds evidence that module DGM-5 is not an artifact. A recent microarray study ${ }^{56}$ did not replicate individual gene effects found in the Mostafavi RNA-Seq study. However, their meta-analysis of the $p$ values identified six genes that showed a consistent effect (the genes had $p<0.05$ in both studies) ${ }^{56}$. Similarly, Leday et $\mathrm{al}^{57}$ used a Bayesian technique to identify concordant gene effects across two independent cohorts. DGM-5, in the current study, contains many genes that are biologically relevant or previously associated with mood disorders (Table 2). However, the pathway enrichment signals of DGM-5, such as apoptosis, point to genes outside of Table 2 as playing an important role in MDD etiology. Incorporating eQTL analysis may fill in part of the functional gaps in the DGM-5 network and further characterize the mechanisms of this mood disorder module.

In the first stage of our analysis, we used MADRS as the primary outcome with the aim of including greater 
Table 2 Mood disorder-related genes in significant modules and summary of their relevance to mood disorders from the literature

\begin{tabular}{|c|c|c|c|}
\hline Module & Gene & Description/related pathways & $\begin{array}{l}\text { Prior studies linkage to mood disorder/ } \\
\text { schizophrenia }\end{array}$ \\
\hline DGM-17 & OPRM1 & $\mu$-opioid receptor/GABAergic synapse & $\begin{array}{l}\text { Stressful life events }{ }^{50} \\
\text { Sustained sadness condition in women }{ }^{51} \\
\text { Response to antidepressants }\end{array}$ \\
\hline DGM-5 & HDAC5 & Histone deacetylase 5/ phospholipase-C Pathway & $\begin{array}{l}\text { MDD pathophysiology } y^{39} \\
\text { Histone pathways }^{66}\end{array}$ \\
\hline DGM-5 & CREB1 & $\begin{array}{l}\text { The cyclic AMP response element-binding protein 1, sequence-specific DNA } \\
\text { binding and enzyme binding/constitutive signaling by AKT1 E17K in cancer }\end{array}$ & $\begin{array}{l}\text { MDD pathophysiology }{ }^{39} \\
\text { Anger expression and treatment outcome in } \\
\text { MDD patients }{ }^{41,42} \\
\text { Gender-specific susceptibility for MDD }{ }^{43,44} \\
\text { Important targets of antidepressants }\end{array}$ \\
\hline DGM-5 & FOXP3 & forkhead box P3, the marker for regulatory $T$ cells/Th2 differentiation pathway & $\begin{array}{l}\text { Decreased expression level in depressed } \\
\text { patients }^{46} \\
\text { Immune system responses }^{40}\end{array}$ \\
\hline DGM-5 & FAS & $\begin{array}{l}\text { fas cell surface death receptor, T-cell activation and apoptosis/ bacterial infections } \\
\text { in CF airways, allograft rejection }\end{array}$ & $\begin{array}{l}\text { Antidepressant prognosis }{ }^{48} \\
\text { Expression increase in depressed patients }{ }^{49}\end{array}$ \\
\hline DGM-5 & FKBP4 & $\begin{array}{l}\text { FK506 Binding Protein 4, paralog of FKBP5/ PEDF induced signaling, HSF1- } \\
\text { dependent transactivation }\end{array}$ & $\begin{array}{l}\text { FKBP5: strong evidence for association with } \\
M D^{67-72}\end{array}$ \\
\hline DGM-5 & AKT1 & $\begin{array}{l}\text { AKT serine/threonine kinase 1, critical mediator of growth factor-induced neuronal } \\
\text { survival/ ICOS-ICosL pathway in T-helper cell, development IGF-1 receptor signaling }\end{array}$ & $\begin{array}{l}\text { Schizophrenia }{ }^{73-75} \\
\text { Depression in different populations }{ }^{76} \\
\text { Neuronal pathways }^{66}\end{array}$ \\
\hline DGM-17 & VRK2 & Vaccinia related Kinase 2/nuclear envelope reassembly, mitotic prophase. & Schizophrenia ${ }^{77-79}$ \\
\hline DGM-17 & TCF7L2 & Transcription Factor 7 Like 2/Wnt signaling pathway & $\begin{array}{l}\text { Schizophrenia }^{80} \\
\text { Genetic variants that are crucial in MDD } \\
\text { susceptibility }^{81}\end{array}$ \\
\hline
\end{tabular}

Table 3 Reactome pathway enrichment results of the two statistically significant MDD modules DGM-5 (replicated) and DGM-17

\begin{tabular}{lllll}
\hline REACTOME pathways & Genes in pathway & $\boldsymbol{p}$ value & FDR $\boldsymbol{q}$ value & Over lapping genes \\
\hline DGM-5: 291 genes & & & & \\
Apoptosis & 148 & $1.19 \mathrm{e}-3$ & 0.108 & AKT1, BAD, PSMD5, PSMD7, FAS \\
Downstream signaling by B cell receptor & 97 & $5.76 \mathrm{e}-4$ & 0.108 & AKT1, BAD, CREB1, PSMD5, PSMD7 \\
PIP3/AKT and PI3K/AKT signaling activation & 29 & $4.82 \mathrm{e}-4$ & 0.108 & AKT1, BAD, CREB1 \\
GAB1 signalosome & 38 & $1.07 \mathrm{e}-3$ & 0.108 & AKT1, BAD, CREB1 \\
PI3K events in ERBB4 and ERBB2 signaling & 38 & $1.07 \mathrm{e}-3$ & 0.108 & AKT1, BAD, CREB1 \\
tRNA aminoacylation & 42 & $1.44 \mathrm{e}-3$ & 0.108 & WARS2, DARS2, LARS \\
AKT phosphorylates targets in the cytosol & 12 & $1.77 \mathrm{e}-3$ & 0.108 & AKT1, BAD \\
DGM-17: 109 genes & & & & N.38e-5 \\
Interactions of Vpr with host cellular proteins & 33 & 0.016 & NUP214, SLC25A5, PSIP1 \\
\hline
\end{tabular}


phenotypic variation than the diagnostic phenotype. Using a precision quantitative trait in a population has the advantage of capturing more variation than a case-control phenotype and may also have more power. For example, it has been shown that dichotomizing a trait variable at the median reduces the power by the same amount as throwing away $1 / 3$ of the data ${ }^{58}$. One limitation of treating MADRS quantitatively is the lack of variation in the scale among healthy subjects. In the second stage analysis, we used diagnostic status as the dependent variable because depression severity was not available.

Another potential limitation of our study is the use of gene expression from PBMCs, which contain many cell types and may not detect brain-specific mechanisms ${ }^{16,56}$. Peripheral blood is an easily accessible source of cells that may more easily translate into a clinical biomarker compared to cell or tissue-specific gene expression. We did not find significant overlap between our top module genes and known cell-type signatures; however, depressionassociated changes in cell frequencies may account for some of the differences in gene expression ${ }^{59}$. While effect sizes may be diluted if small subsets of cells contribute to the signal, we were able to detect module-level signals that replicated in an independent study. Deconvolution methods may help identify cell-specific differences in major immune system cells (such as monocytes, Thelper, $B$ and NK cells) and uncover cell-specific gene expression changes associated with mood disorder phenotypes ${ }^{60}$.

Our modular approach aggregates the effects of genes with shared variation to discover depression gene modules. This approach is influenced by GWAS studies showing that individual variants with small effect sizes, dispersed throughout the genome, drive complex disease risk by key genes and regulatory pathways ${ }^{61}$. Methods for aggregating genetic variation and association signals from prior biological knowledge have been used in GWAS to facilitate more powerful analysis ${ }^{62,63}$. At the level of gene expression, we aggregate the variation from co-expressed genes into modules. This aggregation was done without prior pathway knowledge and in an unbiased way (not conditioned on the phenotype) to mitigate multiple hypothesis testing. Future studies to refine and characterize these depression-related modules will involve identifying regulatory variants through cis- and transeQTL and interaction QTL analysis ${ }^{64}$.

Our analysis used stranded RNA-Seq preprocessing where the forward direction was used for the second fast sequence files. This stranded preprocessing enriches for antisense non-coding RNA, sometimes called Natural Antisense Transcripts (NATs). These NATs are labeled with AS1 (for antisense) appended to their gene symbols, and they are known to recruit epigenetic machinery and other mechanisms to regulate coding RNA (mRNA/ genes). In addition to NATs, stranded preprocessing enriches for protein coding genes that can be transcribed in the antisense direction, which occurs for a significant proportion of mammalian genes (i.e., protein coding). Thus, the replicated module (M5) contains genes that are enriched for antisense expression of protein coding genes and expression of NATs that regulate partner coding genes through an antisense mechanism. We include the RNA-Seq data preprocessed for both antisense RNA and sense RNA gene expression in the github repository (https:/github.com/insilico/DepressionGeneModules).

\section{Code availability \\ https://github.com/insilico/DepressionGeneModules}

\begin{abstract}
Acknowledgements
This study was supported by the National Institute of Mental Health award R01MH098099. The authors thank all staff members at Laureate Institute for Brain Research, especially Brent Wurfel, M.D., Matthew Meyer, M.D. and William Yates, M.D. for conducting psychiatric interviews, Tim Collins for administering clinical interview and assessments, and Megan Cole, Saman Aziz and Cindy Bonebright for performing blood draws. In addition, the authors acknowledge the contributions of Ashlee Taylor, Brenda Davis, Chibing Tan and Debbie Neal from the laboratory of TKT toward the transport, processing, and handling of the blood samples. Finally, we thank staff members at Oklahoma Medical Research Foundation, for generating RNA-Seq data.
\end{abstract}

\section{Author details}

${ }^{1}$ Department of Mathematics, The University of Tulsa, Tulsa, OK, USA. ${ }^{2}$ Laureate Institute for Brain Research, Tulsa, OK, USA. ${ }^{3}$ School of Community Medicine, University of Tulsa, Tulsa, OK, USA. ${ }^{4}$ Department of Educational Psychology, University of Nebraska-Lincoln, Lincoln, NE, USA. ${ }^{5}$ Departments of Surgery and Psychiatry, University of Oklahoma School of Community Medicine, Tulsa, OK, USA. 'Department of Pharmaceutical Sciences, University of Oklahoma College of Pharmacy, Tulsa, OK, USA. ${ }^{7}$ Department of Biochemistry and Microbiology, Oklahoma State University Center for the Health Sciences, Tulsa, OK, USA.

${ }^{8}$ Tandy School of Computer Sciences, The University of Tulsa, Tulsa, OK, USA. ${ }^{9}$ Department of Surgery, Integrative Immunology Center, University of Oklahoma School of Community Medicine, Tulsa, OK, USA. ${ }^{10}$ Arthritis and Clinical Immunology Research Program, Division of Genomics and Data Sciences, Oklahoma Medical Research Foundation, Oklahoma City, OK, USA. ${ }^{11}$ Janssen Research \& Development, LLC, Johnson \& Johnson, Inc, Titusville, NJ, USA. ${ }^{2}$ Stephenson School of Biomedical Engineering, University of Oklahoma, Norman, OK, USA

Conflict of interest

W.C.D. is an employee of Janssen Research \& Development, LLC, of Johnson \& Johnson, Inc. The remaining authors declare that they have no conflict of interests.

\section{Publisher's note}

Springer Nature remains neutral with regard to jurisdictional claims in published maps and institutional affiliations.

Supplementary Information accompanies this paper at (https://doi.org/ 10.1038/s41398-018-0234-3).

Received: 27 November 2017 Revised: 21 June 2018 Accepted: 14 July 2018 Published online: 05 September 2018

\footnotetext{
References

1. Satake, W. et al. Genome-wide association study identifies common variants at four loci as genetic risk factors for Parkinson's disease. Nat. Genet. 41, 1303-1307 (2009).
} 
2. Satoh, J. et al. Microarray analysis identifies an aberrant expression of apoptosis and DNA damage-regulatory genes in multiple sclerosis. Neurobiol. Dis. 18, 537-550 (2005).

3. McKinney, B. A. et al. ReliefSeq: a gene-wise adaptive-K nearest-neighbor feature selection tool for finding gene-gene interactions and main effects in mRNA-Seq gene expression data. PLOS ONE 8, e81527 (2013).

4. Wang, X., Dalkic, E., Wu, M. \& Chan, C. Gene module level analysis: identification to networks and dynamics. Curr. Opin. Biotechnol. 19, 482-491 (2008).

5. Gaiteri, C., Ding, Y., French, B., Tseng, G. C. \& Sibille, E. Beyond modules and hubs: the potential of gene coexpression networks for investigating molecular mechanisms of complex brain disorders. Genes Brain Behav. 13 13-24 (2014).

6. Roy, S., Bhattacharyya, D. K. \& Kalita, J. K. Reconstruction of gene co-expression network from microarray data using local expression patterns. BMC Bioinformatics 15, S10 (2014).

7. Chen, C. et al. Two gene co-expression modules differentiate psychotics and controls. Mol. Psychiatry 18, 1308-1314 (2013).

8. de Jong, S. et al. A gene co-expression network in whole blood of schizophrenia patients is independent of antipsychotic-use and enriched for brainexpressed genes. PLOS ONE 7, e39498 (2012).

9. Torkamani, A., Dean, B., Schork, N. J. \& Thomas, E. A. Coexpression network analysis of neural tissue reveals perturbations in developmental processes in schizophrenia. Genome Res. 20, 403-412 (2010).

10. Voineagu, I. et al. Transcriptomic analysis of autistic brain reveals convergent molecular pathology. Nature 474, 380-384 (2011).

11. Miller, J. A., Oldham, M. C. \& Geschwind, D. H. A systems level analysis of transcriptional changes in Alzheimer's disease and normal aging. J. Neurosci. 28, 1410-1420 (2008).

12. Chang, L. C. et al. A conserved BDNF, glutamate- and GABA-enriched gene module related to human depression identified by coexpression metaanalysis and DNA variant genome-wide association studies. PLOS ONE 9, e90980 (2014).

13. Levinson, D. F. et al. Genetic studies of major depressive disorder: why are there no genome-wide association study findings and what can we do about it? Biol. Psychiatry 76, 510-512 (2014).

14. Hyde, C. L. et al. Identification of 15 genetic loci associated with risk of major depression in individuals of European descent. Nat. Genet. 48, 1031-1036 (2016).

15. Wray N. R. \& Sullivan P. F. Genome-wide association analyses identify 44 risk variants and refine the genetic architecture of major depression. Nat. Genet. 50, 668-681 (2018).

16. Mostafavi, S. et al. Type I interferon signaling genes in recurrent major depression: increased expression detected by whole-blood RNA sequencing. Mol. Psychiatry 19, 1267-1274 (2014).

17. Girvan, M. \& Newman, M. E. Community structure in social and biological networks. Proc. Natl Acad. Sci. USA 99, 7821-7826 (2002).

18. Obayashi, T. et al. COXPRESdb: a database of coexpressed gene networks in mammals. Nucleic Acids Res. 36, D77-D82 (2008).

19. Oldham, M. C., Horvath, S. \& Geschwind, D. H. Conservation and evolution of gene coexpression networks in human and chimpanzee brains. Proc. Nat Acad. Sci. USA 103, 17973-17978 (2006).

20. Langfelder, P. \& Horvath, S. WGCNA: an R package for weighted correlation network analysis. BMC Bioinformatics 9, 559 (2008).

21. Hanzelmann, S., Castelo, R. \& Guinney, J. GSVA: gene set variation analysis for microarray and RNA-seq data. BMC Bioinformatics 14, 7 (2013).

22. Barbie, D. A. et al. Systematic RNA interference reveals that oncogenic KRASdriven cancers require TBK1. Nature 462, 108-112 (2009).

23. FDA. Description of the Hamilton Depression Rating Scale (HAMD) and the Montgomery-Asberg Depression Rating Scale (MADRS) (FDA, USA, 2007).

24. Hansen, K. D., Irizarry, R. A. \& Wu, Z. Removing technical variability in RNAseq data using conditional quantile normalization. Biostatistics 13, 204-216 (2012).

25. ACM. Angle-Based Outlier Detection In High-dimensional Data (ACM, Las Vegas, 2008).

26. Ritchie, M. E. et al. limma powers differential expression analyses for RNAsequencing and microarray studies. Nucleic Acids Res. 43, e47 (2015).

27. Mclntyre, L. M. et al. RNA-seq: technical variability and sampling. BMC Genomics 12, 293 (2011).

28. Rahmani, B. et al. Recursive indirect-paths modularity (RIP-M) for detecting community structure in RNA-seq co-expression networks. Front. Genet. 7, 80 (2016).
29. Boutros, P. C. \& Okey, A. B. Unsupervised pattern recognition: an introduction to the whys and wherefores of clustering microarray data. Brief Bioinformatics 6, 331-343 (2005).

30. Wasserman S. \& Faust K. Social Network Analysis: Methods and Applications. 825 (Cambridge University Press, Cambridge, New York, 1994).

31. Park, S. \& Romer, D. Associations between smoking and depression in adolescence: an integrative review. Taehan Kanho Hakhoe chi 37, 227-241 (2007).

32. Landi, M. T. et al. Gene expression signature of cigarette smoking and its role in lung adenocarcinoma development and survival. PloS ONE 3, e1651 (2008).

33. Besingi, W. \& Johansson, A. Smoke-related DNA methylation changes in the etiology of human disease. Hum. Mol. Genet. 23, 2290-2297 (2014).

34. Benjamini, Y., Drai, D., Elmer, G., Kafkafi, N. \& Golani, I. Controlling the false discovery rate in behavior genetics research. Behav. Brain Res. 125, 279-284 (2001).

35. Ben-Ari Fuchs, S. et al. GeneAnalytics: an integrative gene set analysis tool for next generation sequencing, RNAseq and microarray data. OMICS 20, 139-151 (2016).

36. Stelzer, G. et al. VarElect: the phenotype-based variation prioritizer of the GeneCards Suite. BMC Genomics 17(Suppl 2), 444 (2016).

37. Farber, C. R. Identification of a gene module associated with BMD through the integration of network analysis and genome-wide association data. J. Bone Miner. Res. 25, 2359-2367 (2010).

38. Gold, P. W. The organization of the stress system and its dysregulation in depressive illness. Mol. Psychiatry 20, 32-47 (2015).

39. Iga, J. et al. Altered HDAC5 and CREB mRNA expressions in the peripheral leukocytes of major depression. Prog. Neuropsychopharmacol. Biol. Psychiatry 31, 628-632 (2007).

40. Network, Pathway Analysis Subgroup of the Psychiatric Genomics C. Corrigendum: psychiatric genome-wide association study analyses implicate neuronal, immune and histone pathways. Nat. Neurosci. 18, 1861 (2015).

41. Serretti, A. et al. A preliminary investigation of the influence of CREB1 gene on treatment resistance in major depression. J. Affect Disord. 128, 56-63 (2011).

42. Perlis, R. H. et al. Clinical and genetic dissection of anger expression and CREB1 polymorphisms in major depressive disorder. Biol. Psychiatry $6 \mathbf{2}$, 536-540 (2007)

43. Zubenko, G. S. et al. Sequence variations in CREB1 cosegregate with depressive disorders in women. Mol. Psychiatry 8, 611-618 (2003).

44. Zubenko, G. S. et al. Genetic linkage of region containing the CREB1 gene to depressive disorders in women from families with recurrent, early-onset, major depression. Am. J. Med. Genet. 114, 980-987 (2002).

45. West, A. E., Griffith, E. C. \& Greenberg, M. E. Regulation of transcription factors by neuronal activity. Nat. Rev. Neurosci. 3, 921-931 (2002).

46. Li, Y. et al. Altered expression of CD4 $(+) \operatorname{CD} 25(+)$ regulatory T cells and its 5HT(1a) receptor in patients with major depression disorder. J. Affect Disord. 124, 68-75 (2010)

47. Suzuki, $H$. et al. Altered populations of natural killer cells, cytotoxic T lymphocytes, and regulatory $T$ cells in major depressive disorder: association with sleep disturbance. Brain Behav. Immun. 66, 193-200 (2017).

48. Santos, M. et al. FAS $-670 A>G$ genetic polymorphism is associated with treatment resistant depression. J. Affect Disord. 185, 164-169 (2015).

49. Szuster-Ciesielska, A. et al. Accelerated apoptosis of blood leukocytes and oxidative stress in blood of patients with major depression. Prog. Neuropsychopharmacol. Biol. Psychiatry 32, 686-694 (2008).

50. Swann, G. et al. Effect of OPRM1 and stressful life events on symptoms of major depression in African American adolescents. J. Affect Disord. 162, 12-19 (2014).

51. Kennedy, S. E., Koeppe, R. A., Young, E. A. \& Zubieta, J. K. Dysregulation of endogenous opioid emotion regulation circuitry in major depression in women. Arch. Gen. Psychiatry 63, 1199-1208 (2006).

52. McKinnon, M. C., Yucel, K., Nazarov, A. \& MacQueen, G. M. A meta-analysis examining clinical predictors of hippocampal volume in patients with major depressive disorder. J. Psychiatry Neurosci. 34, 41-54 (2009).

53. Eilat, E., Mendlovic, S., Doron, A., Zakuth, V. \& Spirer, Z. Increased apoptosis in patients with major depression: a preliminary study. J. Immunol. 163, 533-534 (1999).

54. Kaur, S. et al. Role of the Akt pathway in mRNA translation of interferonstimulated genes. Proc. Natl Acad. Sci. USA 105, 4808-4813 (2008).

55. Langfelder, P., Mischel, P. S. \& Horvath, S. When is hub gene selection better than standard meta-analysis? PLOS ONE 8, e61505 (2013).

56. Jansen, R. et al. Gene expression in major depressive disorder. Mol. Psychiatry 21, 444 (2016). 
57. Leday, G. G. R. et al. replicable and coupled changes in innate and adaptive immune gene expression in two case-control studies of blood microarrays in major depressive disorder. Biol. Psychiatry 83, 70-80 (2017).

58. Altman, D. G. \& Royston, P. The cost of dichotomising continuous variables. BMJ 332, 1080 (2006).

59. Padmos, R. C. et al. A discriminating messenger RNA signature for bipolar disorder formed by an aberrant expression of inflammatory genes in monocytes. Arch. Gen. Psychiatry 65, 395-407 (2008).

60. Gaujoux, R. \& Seoighe, C. CellMix: a comprehensive toolbox for gene expression deconvolution. Bioinformatics 29, 2211-2212 (2013).

61. Furlong, L. I. Human diseases through the lens of network biology. Trends Genet. 29, 150-159 (2013).

62. Wang, K. Li, M. \& Hakonarson, H. Analysing biological pathways in genomewide association studies. Nat. Rev. Genet. 11, 843-854 (2010).

63. McKinney, B. A. et al. The integration of epistasis network and functional interactions in a GWAS implicates RXR pathway genes in the immune response to smallpox vaccine. PLoS ONE 11, e0158016 (2016).

64. Lareau, C. A. et al. An interaction quantitative trait loci tool implicates epistatic functional variants in an apoptosis pathway in smallpox vaccine eQTL data. Genes Immun. 17, 244-250 (2016).

65. Garriock, H. A. et al. Association of mu-opioid receptor variants and response to citalopram treatment in major depressive disorder. Am. J. Psychiatry $\mathbf{1 6 7}$ 565-573 (2010).

66. Network, Pathway Analysis Subgroup of Psychiatric Genomics C. Psychiatric genome-wide association study analyses implicate neuronal, immune and histone pathways. Nat. Neurosci. 18, 199-209 (2015).

67. Tatro, E. T., Everall, I. P., Kaul, M. \& Achim, C. L. Modulation of glucocorticoid receptor nuclear translocation in neurons by immunophilins FKBP51 and FKBP52: implications for major depressive disorder. Brain Res. 1286, 1-12 (2009).

68. Binder, E. B. et al. Polymorphisms in FKBP5 are associated with increased recurrence of depressive episodes and rapid response to antidepressant treatment. Nat. Genet. 36, 1319-1325 (2004).

69. Ellsworth, K. A. et al. FKBP5 genetic variation: association with selective serotonin reuptake inhibitor treatment outcomes in major depressive disorder. Pharm. Genomics 23, 156-166 (2013).
70. Scheuer, S. et al. FKBP5 polymorphisms moderate the influence of adverse life events on the risk of anxiety and depressive disorders in preschool children J. Psychiatr. Res. 72, 30-36 (2016).

71. Lavebratt, C., Aberg, E., Sjoholm, L. K. \& Forsell, Y. Variations in FKBP5 and BDNF genes are suggestively associated with depression in a Swedish population-based cohort. J. Affect Disord. 125, 249-255 (2010)

72. Tatro, E. T. et al. Differential expression of immunophilins FKBP51 and FKBP52 in the frontal cortex of HIV-infected patients with major depressive disorder. J. Neuroimmune Pharmacol. 4, 218-226 (2009).

73. Emamian, E. S., Hall, D., Birnbaum, M. J., Karayiorgou, M. \& Gogos, J. A. Convergent evidence for impaired AKT1-GSK3beta signaling in schizophrenia. Nat. Genet. 36, 131-137 (2004)

74. Mathur, A., Law, M. H., Megson, I. L., Shaw, D. J. \& Wei, J. Genetic association of the AKT1 gene with schizophrenia in a British population. Psychiatr. Genet. 20, 118-122 (2010).

75. Xu, M. Q. et al. Association of AKT1 gene polymorphisms with risk of schizophrenia and with response to antipsychotics in the Chinese population. J. Clin. Psychiatry 68, 1358-1367 (2007).

76. Pereira, P. A. et al. Genetic variant of AKT1 and AKTIP associated with late-onset depression in a Brazilian population. Int. J. Geriatr. Psychiatry 29, 399-405 (2014).

77. Li, M. et al. Meta-analysis and brain imaging data support the involvement of VRK2 (rs2312147) in schizophrenia susceptibility. Schizophr. Res. 142, 200-205 (2012).

78. Schizophrenia Working Group of the Psychiatric Genomics C. Biological insights from 108 schizophrenia-associated genetic loci. Nature 511, 421-427 (2014).

79. Zhang, B. et al. Association of the VRK2 gene rs3732136 polymorphism with schizophrenia in a Northwest Chinese Han population. Genet. Mol. Res. 14 9404-9411 (2015).

80. Stefansson, $\mathrm{H}$. et al. Common variants conferring risk of schizophrenia. Nature 460, 744-747 (2009).

81. Inkster, B. et al. Pathway-based approaches to imaging genetics association studies: Wnt signaling, GSK3beta substrates and major depression. Neuroimage 53, 908-917 (2010). 